\title{
Exact Solutions for Some Fractional Partial Differential Equations by the $\left(G^{\prime} / G\right)$ Method
}

\author{
Bin Zheng \\ School of Science, Shandong University of Technology, Zibo, Shandong 255049, China \\ Correspondence should be addressed to Bin Zheng; zhengbin2601@126.com
}

Received 19 July 2013; Accepted 23 September 2013

Academic Editor: Rasajit Bera

Copyright (C) 2013 Bin Zheng. This is an open access article distributed under the Creative Commons Attribution License, which permits unrestricted use, distribution, and reproduction in any medium, provided the original work is properly cited.

We apply the $\left(G^{\prime} / G\right)$ method to seek exact solutions for several fractional partial differential equations including the space-time fractional $(2+1)$-dimensional dispersive long wave equations, the $(2+1)$-dimensional space-time fractional Nizhnik-NovikovVeselov system, and the time fractional fifth-order Sawada-Kotera equation. The fractional derivative is defined in the sense of modified Riemann-liouville derivative. Based on a certain variable transformation, these fractional partial differential equations are transformed into ordinary differential equations of integer order. With the aid of mathematical software, a variety of exact solutions for them are obtained.

\section{Introduction}

In recent decades, fractional differential equations have gained much attention as they are widely used to describe various complex phenomena in many fields such as the fluid flow, signal processing, control theory, systems identification, biology, and other areas. Among the investigations for fractional differential equations, research for seeking exact solutions and approximate solutions of fractional differential equations is a hot topic. Many powerful and efficient methods have been proposed so far, for example, the fractional variational iteration method [1-5], the Adomian's decomposition method [6-8], the homotopy perturbation method [9], the finite difference method [10], the finite element method [11], the fractional subequation method [12-14], and so on. Using these methods, solutions with various forms for some given fractional differential equations have been established.

In this paper, we will apply the $\left(G^{\prime} / G\right)$ method [1521] for solving some fractional partial differential equations in the sense of modified Riemann-Liouville derivative by Jumarie. The definition and some important properties for the Jumarie's modified Riemann-Liouville derivative of order $\alpha$ are listed as follows [22]:

$$
\begin{gathered}
D_{t}^{\alpha} f(t)= \begin{cases}\frac{1}{\Gamma(1-\alpha)} \frac{d}{d t} \\
\times \int_{0}^{t}(t-\xi)^{-\alpha} \\
\times(f(\xi)-f(0)) d \xi, & 0<\alpha<1, \\
\left(f^{(n)}(t)\right)^{(\alpha-n)}, & n \leq \alpha<n+1, n \geq 1,\end{cases} \\
D_{t}^{\alpha} t^{r}=\frac{\Gamma(1+r)}{\Gamma(1+r-\alpha)} t^{r-\alpha}, \\
D_{t}^{\alpha}(f(t) g(t))=g(t) D_{t}^{\alpha} f(t)+f(t) D_{t}^{\alpha} g(t), \\
D_{t}^{\alpha} f[g(t)]=f_{g}^{\prime}[g(t)] D_{t}^{\alpha} g(t)=D_{g}^{\alpha} f[g(t)]\left(g^{\prime}(t)\right)^{\alpha} .
\end{gathered}
$$

The rest of this paper is organized as follows. In Section 2, we give the description of the $\left(G^{\prime} / G\right)$ method for solving fractional partial differential equations. Then in Section 3 we apply this method to establish exact solutions for the space-time fractional $(2+1)$-dimensional dispersive long wave equations, the space-time fractional $(2+1)$-dimensional 
Nizhnik-Novikov-Veselov system, and the time fractional fifth-order Sawada-Kotera equation. Some conclusions are presented at the end of the paper.

\section{Description of the $\left(G^{\prime} / G\right)$ Method for Fractional Partial Differential Equations}

In this section we give the description of the $\left(G^{\prime} / G\right)$ method for solving fractional partial differential equations.

Suppose that a fractional partial differential equation, say in the independent variables $t, x_{1}, x_{2}, \ldots, x_{n}$, is given by

$$
\begin{array}{r}
P\left(u_{1}, \ldots, u_{k}, D_{t}^{\alpha} u_{1}, \ldots, D_{t}^{\alpha} u_{k}, D_{x_{1}}^{\beta} u_{1}, \ldots,\right. \\
\left.D_{x_{1}}^{\beta} u_{k}, \ldots, D_{x_{n}}^{\gamma} u_{1}, \ldots, D_{x_{n}}^{\gamma} u_{k}, \ldots\right)=0,
\end{array}
$$

where $u_{i}=u_{i}\left(t, x_{1}, x_{2}, \ldots, x_{n}\right), i=1, \ldots, k$, are unknown functions and $P$ is a polynomial in $u_{i}$ and their various partial derivatives including fractional derivatives.

Step 1. Execute a certain variable transformation:

$$
u_{i}\left(t, x_{1}, x_{2}, \ldots, x_{n}\right)=U_{i}(\xi), \quad \xi=\xi\left(t, x_{1}, x_{2}, \ldots, x_{n}\right)
$$

such that (4) can be turned into the following ordinary differential equation of integer order with respect to the variable $\xi$ :

$$
\widetilde{P}\left(U_{1}, \ldots, U_{k}, U_{1}^{\prime}, \ldots, U_{k}^{\prime}, U_{1}^{\prime \prime}, \ldots, U_{k}^{\prime \prime}, \ldots\right)=0
$$

Step 2. Suppose that the solution of (6) can be expressed by a polynomial in $\left(G^{\prime} / G\right)$ as follows:

$$
U_{j}(\xi)=\sum_{i=0}^{m_{j}} a_{j, i}\left(\frac{G^{\prime}}{G}\right)^{i}, \quad j=1,2, \ldots, k
$$

where $G=G(\xi)$ satisfies the second-order ODE in the form

$$
G^{\prime \prime}+\lambda G^{\prime}+\mu G=0
$$

and $\lambda, \mu, a_{j, i}, i=0,1, \ldots, m_{j}, j=1,2, \ldots, k$, are constants to be determined later, $a_{j, m} \neq 0$. The positive integer $m_{j}$ can be determined by considering the homogeneous balance between the highest order derivatives and nonlinear terms appearing in (6).
By the generalized solutions of (8), we have

$$
\begin{aligned}
& \begin{array}{l}
-\frac{\lambda}{2}+\frac{\sqrt{\lambda^{2}-4 \mu}}{2} \\
\times\left(\left(\begin{array}{c}
C_{1} \sinh \frac{\sqrt{\lambda^{2}-4 \mu}}{2} \xi \\
\left.+C_{2} \cosh \frac{\sqrt{\lambda^{2}-4 \mu}}{2} \xi\right)
\end{array}\right)\right.
\end{array} \\
& \times\left(C_{1} \cosh \frac{\sqrt{\lambda^{2}-4 \mu}}{2} \xi\right. \\
& \left.\left.+C_{2} \sinh \frac{\sqrt{\lambda^{2}-4 \mu}}{2} \xi\right)^{-1}\right), \quad \lambda^{2}-4 \mu>0, \\
& \left(\frac{G^{\prime}}{G}\right)=\left\{\begin{array}{l}
\lambda-\frac{\sqrt{4 \mu-\lambda^{2}}}{2}+\frac{\sqrt{2}}{}
\end{array}\right. \\
& \times\left(\left(-C_{1} \sin \frac{\sqrt{4 \mu-\lambda^{2}}}{2} \xi\right.\right. \\
& \left.+C_{2} \cos \frac{\sqrt{4 \mu-\lambda^{2}}}{2} \xi\right) \\
& \times\left(C_{1} \cos \frac{\sqrt{4 \mu-\lambda^{2}}}{2} \xi\right. \\
& \left.\left.+C_{2} \sin \frac{\sqrt{4 \mu-\lambda^{2}}}{2} \xi\right)^{-1}\right), \quad \lambda^{2}-4 \mu<0, \\
& -\frac{\lambda}{2}+\frac{C_{2}}{C_{1}+C_{2} \xi}, \\
& \lambda^{2}-4 \mu=0,
\end{aligned}
$$

where $C_{1}$ and $C_{2}$ are arbitrary constants.

Step 3. Substituting (7) into (6) and using (8), collecting all terms with the same order of $\left(G^{\prime} / G\right)$ together, the left-hand side of (6) is converted into another polynomial in $\left(G^{\prime} / G\right)$. Equating each coefficient of this polynomial to zero yields a set of algebraic equations for $\lambda, \mu, a_{j, i}, i=0,1, \ldots, m_{j}, j=$ $1,2, \ldots, k$.

Step 4. Solving the equations system in Step 3 and using (9), we can construct a variety of exact solutions for (4). 


\section{Applications of the Method}

3.1. Space-Time Fractional $(2+1)$-Dimensional Dispersive Long Wave Equations. We consider the following spacetime fractional $(2+1)$-dimensional dispersive long wave equations:

$$
\begin{aligned}
& \frac{\partial^{2 \alpha} u}{\partial y^{\alpha} \partial t^{\alpha}}+\frac{\partial^{2 \alpha} v}{\partial x^{2 \alpha}}+\frac{\partial^{\alpha}\left(u\left(\partial^{\alpha} u / \partial x^{\alpha}\right)\right)}{\partial y^{\alpha}}=0, \\
& \frac{\partial^{\alpha} v}{\partial t^{\alpha}}+\frac{\partial^{\alpha} u}{\partial x^{\alpha}}+\frac{\partial^{\alpha}(u v)}{\partial x^{\alpha}}+\frac{\partial^{3 \alpha} u}{\partial x^{2 \alpha} \partial y^{\alpha}}=0,
\end{aligned}
$$

which are a variation of the known $(2+1)$-dimensional dispersive long wave equations [23-36]:

$$
\begin{gathered}
u_{y t}+v_{x x}+\left(u u_{x}\right)_{y}=0, \\
v_{t}+u_{x}+(u v)_{x}+u_{x x y}=0 .
\end{gathered}
$$

Some types of exact solutions for (11) have been obtained in [23-36] by the use of various methods including the Riccati subequation method [23, 24, 29], the nonlinear transformation method [25], Jacobi function method [27, 28, 36], $\left(G^{\prime} / G\right)$-expansion method [26], modified CK's direct method [30], EXP-function method [31], Hopf-Cole transformation method [32], modified extended Fan's sub-equation method $[33,34]$, and generalized algebraic method [35]. But we notice so far that no research has been paid for (10). In the following we will apply the described method in Section 2 to (10).

To begin with, we suppose $u(x, y, t)=U(\xi), v(x, y, t)=$ $V(\xi)$, where $\xi=(c / \Gamma(1+\alpha)) t^{\alpha}+\left(k_{1} / \Gamma(1+\alpha)\right) x^{\alpha}+\left(k_{2} / \Gamma(1+\right.$ $\alpha) y^{\alpha}+\xi_{0}, k_{1}, k_{2}, c, \xi_{0}$ are all constants with $k_{1}, k_{2}, c \neq 0$. Then by the use of the first equality in (3), we obtain $D_{x}^{\alpha} u=$ $D_{x}^{\alpha} U(\xi)=U^{\prime}(\xi) D_{x}^{\alpha} \xi=k_{1} U^{\prime}(\xi), D_{y}^{\alpha} u=D_{y}^{\alpha} U(\xi)=$ $U^{\prime}(\xi) D_{y}^{\alpha} \xi=k_{2} U^{\prime}(\xi), D_{t}^{\alpha} u=D_{t}^{\alpha} U(\xi)=U^{\prime}(\xi) D_{t}^{\alpha} \xi=c U^{\prime}(\xi)$, and then (10) can be turned into

$$
\begin{gathered}
c k_{2} U^{\prime \prime}+k_{1}^{2} V^{\prime \prime}+k_{1} k_{2}\left(U U^{\prime \prime}+\left(U^{\prime}\right)^{2}\right)=0, \\
c V^{\prime}+k_{1} U^{\prime}+k_{1}(U V)^{\prime}+k_{1}^{2} k_{2} U^{\prime \prime \prime}=0 .
\end{gathered}
$$

Suppose that the solutions of (12), can be expressed by

$$
\begin{aligned}
& U(\xi)=\sum_{i=0}^{m_{1}} a_{i}\left(\frac{G^{\prime}}{G}\right)^{i}, \\
& V(\xi)=\sum_{i=0}^{m_{2}} b_{i}\left(\frac{G^{\prime}}{G}\right)^{i} .
\end{aligned}
$$

Balancing the order of $V^{\prime \prime}$ and $U U^{\prime \prime},(U V)^{\prime}$ and $U^{\prime \prime \prime}$ in (12) we can obtain $m_{1}=1, m_{2}=2$. So we have

$$
\begin{gathered}
U(\xi)=a_{0}+a_{1}\left(\frac{G^{\prime}}{G}\right), \\
V(\xi)=b_{0}+b_{1}\left(\frac{G^{\prime}}{G}\right)+b_{2}\left(\frac{G^{\prime}}{G}\right)^{2} .
\end{gathered}
$$

Substituting (14) into (12), using (8), collecting all the terms with the same power of $\left(G^{\prime} / G\right)$ together, and equating each coefficient to zero, yields a set of algebraic equations. Solving these equations yields

$$
\begin{gathered}
a_{0}=\frac{ \pm k_{1}^{2} \lambda-c}{k_{1}}, \quad a_{1}= \pm 2 k_{1}, \\
b_{0}=-2 k_{1} k_{2} \mu-1, \quad b_{1}=-2 k_{1} k_{2} \lambda, \\
b_{2}=-2 k_{1} k_{2} .
\end{gathered}
$$

Substituting the result above into (14) and combining it with (9) we can obtain the following exact solutions to (10). When $\lambda^{2}-4 \mu>0$,

$$
\begin{aligned}
& u_{1}(x, y, t) \\
& =\frac{ \pm k_{1}^{2} \lambda-c}{k_{1}} \\
& \pm 2 k_{1}\left[-\frac{\lambda}{2}+\frac{\sqrt{\lambda^{2}-4 \mu}}{2}\right. \\
& \times\left(\left(C_{1} \sinh \frac{\sqrt{\lambda^{2}-4 \mu}}{2} \xi\right.\right. \\
& \left.+C_{2} \cosh \frac{\sqrt{\lambda^{2}-4 \mu}}{2} \xi\right) \\
& \times\left(C_{1} \cosh \frac{\sqrt{\lambda^{2}-4 \mu}}{2} \xi\right. \\
& \left.\left.\left.+C_{2} \sinh \frac{\sqrt{\lambda^{2}-4 \mu}}{2} \xi\right)^{-1}\right)\right] \\
& -\frac{3}{2} k_{1}^{2}\left[-\frac{\lambda}{2}+\frac{\sqrt{\lambda^{2}-4 \mu}}{2}\right. \\
& \times\left(\left(C_{1} \sinh \frac{\sqrt{\lambda^{2}-4 \mu}}{2} \xi\right.\right. \\
& \left.+C_{2} \cosh \frac{\sqrt{\lambda^{2}-4 \mu}}{2} \xi\right)
\end{aligned}
$$




$$
\begin{aligned}
& \times\left(C_{1} \cosh \frac{\sqrt{\lambda^{2}-4 \mu}}{2} \xi\right. \\
& \left.\left.\left.+C_{2} \sinh \frac{\sqrt{\lambda^{2}-4 \mu}}{2} \xi\right)^{-1}\right)\right]^{2} \\
& v_{1}(x, y, t) \\
& =-2 k_{1} k_{2} \mu-1-2 k_{1} k_{2} \lambda \\
& \times\left[-\frac{\lambda}{2}+\frac{\sqrt{\lambda^{2}-4 \mu}}{2}\right. \\
& \times\left(\left(C_{1} \sinh \frac{\sqrt{\lambda^{2}-4 \mu}}{2} \xi\right.\right. \\
& \left.+C_{2} \cosh \frac{\sqrt{\lambda^{2}-4 \mu}}{2} \xi\right) \\
& \times\left(C_{1} \cosh \frac{\sqrt{\lambda^{2}-4 \mu}}{2} \xi\right. \\
& \left.\left.\left.+C_{2} \sinh \frac{\sqrt{\lambda^{2}-4 \mu}}{2} \xi\right)^{-1}\right)\right] \\
& -2 k_{1} k_{2}\left[-\frac{\lambda}{2}+\frac{\sqrt{\lambda^{2}-4 \mu}}{2}\right. \\
& \times\left(\left(C_{1} \sinh \frac{\sqrt{\lambda^{2}-4 \mu}}{2} \xi\right.\right. \\
& \left.+C_{2} \cosh \frac{\sqrt{\lambda^{2}-4 \mu}}{2} \xi\right) \\
& \times\left(C_{1} \cosh \frac{\sqrt{\lambda^{2}-4 \mu}}{2} \xi\right. \\
& \left.\left.\left.+C_{2} \sinh \frac{\sqrt{\lambda^{2}-4 \mu}}{2} \xi\right)^{-1}\right)\right]^{2}, \\
& u_{2}(x, y, t) \\
& =\frac{ \pm k_{1}^{2} \lambda-c}{k_{1}} \\
& \pm 2 k_{1}\left[-\frac{\lambda}{2}+\frac{\sqrt{4 \mu-\lambda^{2}}}{2}\right. \\
& \times\left(\left(-C_{1} \sin \frac{\sqrt{4 \mu-\lambda^{2}}}{2} \xi\right.\right. \\
& \left.+C_{2} \cos \frac{\sqrt{4 \mu-\lambda^{2}}}{2} \xi\right) \\
& \times\left(C_{1} \cos \frac{\sqrt{4 \mu-\lambda^{2}}}{2} \xi\right. \\
& \left.\left.\left.+C_{2} \sin \frac{\sqrt{4 \mu-\lambda^{2}}}{2} \xi\right)^{-1}\right)\right] \\
& -\frac{3}{2} k_{1}^{2}\left[-\frac{\lambda}{2}+\frac{\sqrt{4 \mu-\lambda^{2}}}{2}\right. \\
& \times\left(\left(-C_{1} \sin \frac{\sqrt{4 \mu-\lambda^{2}}}{2} \xi\right.\right. \\
& \left.+C_{2} \cos \frac{\sqrt{4 \mu-\lambda^{2}}}{2} \xi\right) \\
& \times\left(C_{1} \cos \frac{\sqrt{4 \mu-\lambda^{2}}}{2} \xi\right. \\
& \left.\left.\left.+C_{2} \sin \frac{\sqrt{4 \mu-\lambda^{2}}}{2} \xi\right)\right)\right]^{2} \\
& v_{2}(x, y, t) \\
& =-2 k_{1} k_{2} \mu-1
\end{aligned}
$$




$$
\begin{aligned}
& -2 k_{1} k_{2} \lambda\left[-\frac{\lambda}{2}+\frac{\sqrt{4 \mu-\lambda^{2}}}{2}\right. \\
& \times\left(\left(-C_{1} \sin \frac{\sqrt{4 \mu-\lambda^{2}}}{2} \xi\right.\right. \\
& \left.+C_{2} \cos \frac{\sqrt{4 \mu-\lambda^{2}}}{2} \xi\right) \\
& \times\left(C_{1} \cos \frac{\sqrt{4 \mu-\lambda^{2}}}{2} \xi\right. \\
& \left.\left.\left.+C_{2} \sin \frac{\sqrt{4 \mu-\lambda^{2}}}{2} \xi\right)^{-1}\right)\right] \\
& -2 k_{1} k_{2}\left[-\frac{\lambda}{2}+\frac{\sqrt{4 \mu-\lambda^{2}}}{2}\right. \\
& \times\left(\left(-C_{1} \sin \frac{\sqrt{4 \mu-\lambda^{2}}}{2} \xi\right.\right. \\
& \left.+C_{2} \cos \frac{\sqrt{4 \mu-\lambda^{2}}}{2} \xi\right) \\
& \times\left(C_{1} \cos \frac{\sqrt{4 \mu-\lambda^{2}}}{2} \xi\right. \\
& \left.\left.\left.+C_{2} \sin \frac{\sqrt{4 \mu-\lambda^{2}}}{2} \xi\right)\right)\right]^{2},
\end{aligned}
$$

where $\xi=(c / \Gamma(1+\alpha)) t^{\alpha}+\left(k_{1} / \Gamma(1+\alpha)\right) x^{\alpha}+\left(k_{2} / \Gamma(1+\alpha)\right) y^{\alpha}+\xi_{0}$.

In Figures 3 and 4, the periodic function solutions (17) are demonstrated with some certain parameters.

When $\lambda^{2}-4 \mu=0$,

$$
\begin{aligned}
u_{3}(x, y, t)= & \frac{ \pm k_{1}^{2} \lambda-c}{k_{1}} \pm 2 k_{1}\left[-\frac{\lambda}{2}+\frac{C_{2}}{C_{1}+C_{2} \xi}\right] \\
& -\frac{3}{2} k_{1}^{2}\left[-\frac{\lambda}{2}+\frac{C_{2}}{C_{1}+C_{2} \xi}\right]^{2},
\end{aligned}
$$

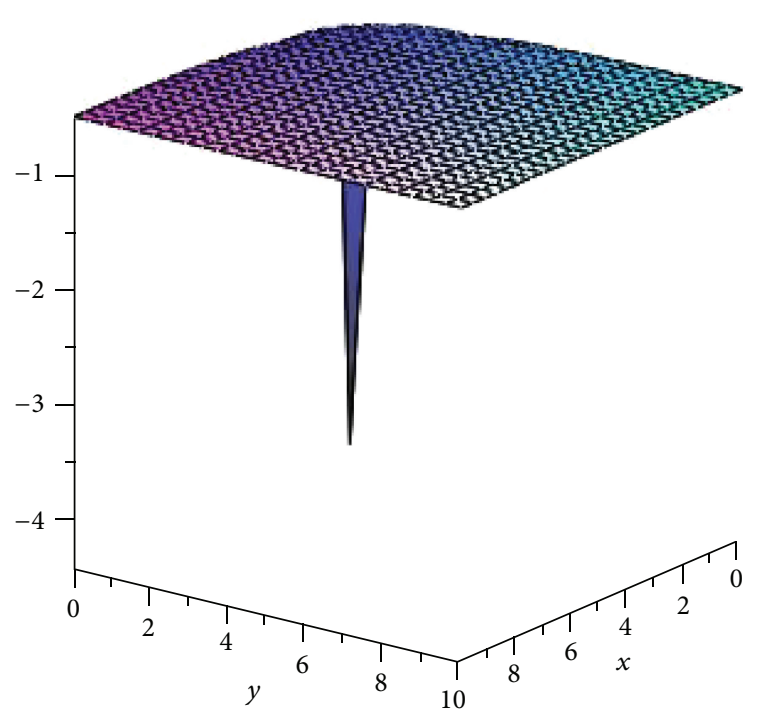

FIGURE 1: The solution $u_{1}$ with the positive symbol selected in \pm and $\lambda=4, \mu=3, c=k_{1}=k_{2}=1, C_{2}=\xi_{0}=0, \alpha=1 / 2$, and $t=0.1$.

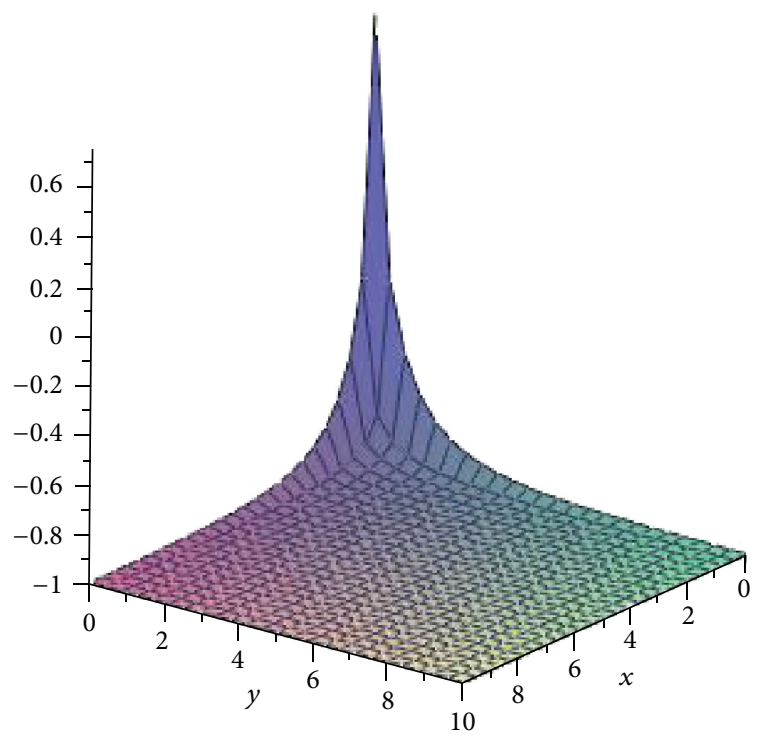

FIgURE 2: The solution $v_{1}$ with $\lambda=4, \mu=3, c=k_{1}=k_{2}=1$, $C_{2}=\xi_{0}=0, \alpha=1 / 2$, and $t=0.1$.

$$
\begin{aligned}
v_{3}(x, y, t)= & -2 k_{1} k_{2} \mu-1 \\
& -2 k_{1} k_{2} \lambda\left[-\frac{\lambda}{2}+\frac{C_{2}}{C_{1}+C_{2} \xi}\right] \\
& -2 k_{1} k_{2}\left[-\frac{\lambda}{2}+\frac{C_{2}}{C_{1}+C_{2} \xi}\right]^{2},
\end{aligned}
$$

where $\xi=(c / \Gamma(1+\alpha)) t^{\alpha}+\left(k_{1} / \Gamma(1+\alpha)\right) x^{\alpha}+\left(k_{2} / \Gamma(1+\alpha)\right) y^{\alpha}+\xi_{0}$. Remark 1. The established solutions in (16)-(18) are new exact solutions for the space-time fractional $(2+1)$ dimensional dispersive long wave equations. 


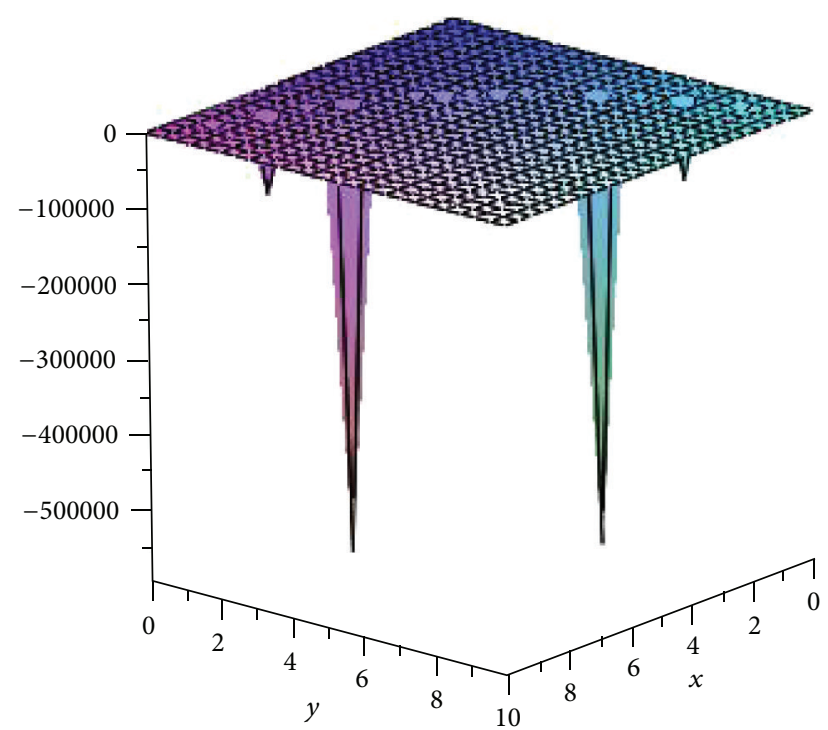

FIGURE 3: The solution $u_{2}$ with the positive symbol selected in \pm and $\lambda=2, \mu=2, c=k_{1}=k_{2}=1, C_{2}=\xi_{0}=0, \alpha=1 / 2$, and $t=0.1$.

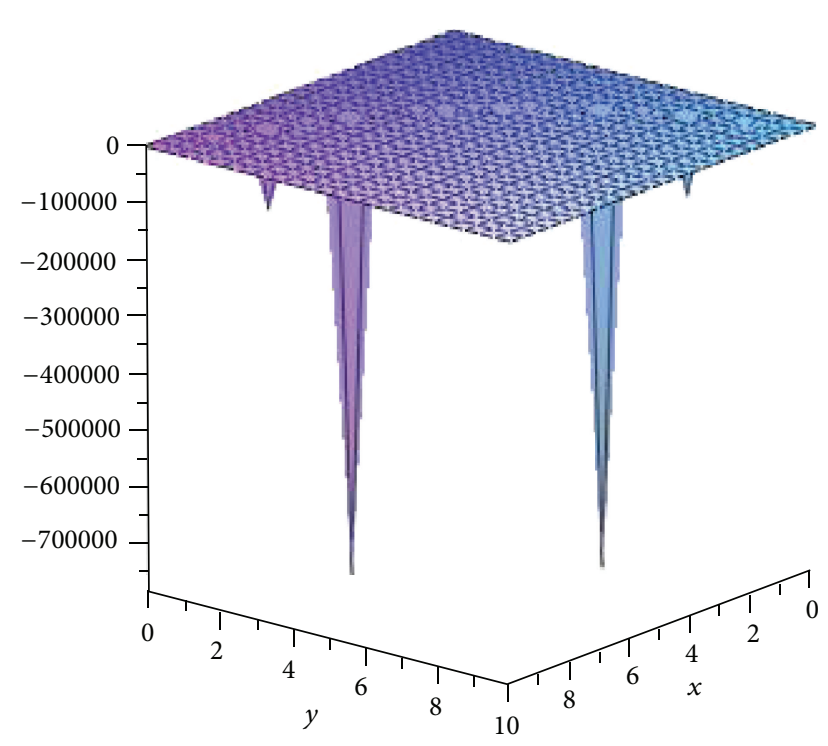

FIGURE 4: The solution $v_{2}$ with $\lambda=2, \mu=2, c=k_{1}=k_{2}=1$, $C_{2}=\xi_{0}=0, \alpha=1 / 2$, and $t=0.1$.

3.2. Space-Time Fractional $(2+1)$-Dimensional NizhnikNovikov-Veselov System. We will consider the space-time fractional $(2+1)$-dimensional Nizhnik-Novikov-Veselov (NNV) system:

$$
\begin{gathered}
D_{t}^{\alpha} u+a D_{x}^{3 \beta} u+b D_{y}^{3 \gamma} u+c D_{x}^{\beta} u+d D_{y}^{\gamma} u \\
=3 a D_{x}^{\beta}(u v)+3 b D_{y}^{\gamma}(u w), \\
D_{x}^{\beta} u=D_{y}^{\gamma} v, \\
D_{y}^{\gamma} u=D_{x}^{\beta} w, \\
0<\alpha, \beta, \gamma \leq 1,
\end{gathered}
$$

which is a variation of the following $(2+1)$-dimensional Nizhnik-Novikov-Veselov (NNV) system [21, 37, 38]:

$$
\begin{gathered}
u_{t}+a u_{x x x}+b u_{y y y}+c u_{x}+d u_{y} \\
=3 a(u v)_{x}+3 b(u w)_{y}, \\
u_{x}=v_{y} \\
u_{y}=w_{x} .
\end{gathered}
$$

Suppose $u(x, y, t)=U(\xi), v(x, y, t)=V(\xi), w(x, y, t)=$ $W(\xi)$, where $\xi=(c / \Gamma(1+\alpha)) t^{\alpha}+(k / \Gamma(1+\beta)) x^{\beta}+(l / \Gamma(1+$ $\gamma)) y^{\gamma}+\xi_{0}, c, k, l, \xi_{0}$ are all constants with $k_{1}, k_{2}, c \neq 0$. Then by the use of the first equality in (3), (19) can be turned into the following forms:

$$
\begin{gathered}
c u^{\prime}+a k^{3} u^{\prime \prime \prime}+b l^{3} u^{\prime \prime \prime}+c k u^{\prime}+d l u^{\prime} \\
=3 a k(u v)^{\prime}+3 b l(u w)^{\prime}, \\
k u^{\prime}=l v^{\prime} \\
l u^{\prime}=k w^{\prime} .
\end{gathered}
$$

Integrating (21) once, we have

$$
\begin{gathered}
c u+a k^{3} u^{\prime \prime}+b l^{3} u^{\prime \prime}+c k u+d l u \\
=3 a k u v+3 b l u w+g_{1}, \\
k u=l v+g_{2}, \\
l u=k w+g_{3},
\end{gathered}
$$

where $g_{1}, g_{2}$, and $g_{3}$ are the integration constants.

Suppose that the solutions of (22) can be expressed by a polynomial in $\left(G^{\prime} / G\right)$ as follows:

$$
\begin{aligned}
& U(\xi)=\sum_{i=0}^{m_{1}} a_{i}\left(\frac{G^{\prime}}{G}\right)^{i}, \\
& V(\xi)=\sum_{i=0}^{m_{2}} b_{i}\left(\frac{G^{\prime}}{G}\right)^{i}, \\
& W(\xi)=\sum_{i=0}^{m_{3}} c_{i}\left(\frac{G^{\prime}}{G}\right)^{i},
\end{aligned}
$$

where $a_{i}, b_{i}$, and $c_{i}$ are constants. Balancing the order of $u^{\prime \prime}$ and $u v$, and the order of $u$ and $v$, the order of $u$ and $w$ in (22), we can obtain $m_{1}=m_{2}=m_{3}=2$. So we have

$$
\begin{aligned}
& U(\xi)=a_{0}+a_{1}\left(\frac{G^{\prime}}{G}\right)^{1}+a_{2}\left(\frac{G^{\prime}}{G}\right)^{2}, \\
& V(\xi)=b_{0}+b_{1}\left(\frac{G^{\prime}}{G}\right)^{1}+b_{2}\left(\frac{G^{\prime}}{G}\right)^{2}, \\
& W(\xi)=c_{0}+c_{1}\left(\frac{G^{\prime}}{G}\right)^{1}+c_{2}\left(\frac{G^{\prime}}{G}\right)^{2} .
\end{aligned}
$$


Substituting (24) into (22), using (8), collecting all the terms with the same power of $\left(G^{\prime} / G\right)$ together, and equating each coefficient to zero, yields a set of algebraic equations. Solving these equations yields.

Case 1. Consider

$$
\begin{gathered}
a_{2}=2 k l, \quad a_{1}=2 k l \lambda, \quad a_{0}=2 k l \mu, \\
b_{2}=2 k^{2}, \quad b_{1}=2 k^{2} \lambda, \quad b_{0}=2 k^{2} \mu, \\
c_{2}=2 l^{2}, \quad c_{1}=2 l^{2} \lambda, \quad c_{0}=2 l^{2} \mu, \\
c=4 a k^{3} \mu-a k^{3} \lambda^{2}-b l^{3} \lambda^{2}-d l+4 b l^{3} \mu-c k, \\
g_{1}=g_{2}=g_{3}=0,
\end{gathered}
$$

where $k$ and $l$ are arbitrary constants.

Substituting the result above into (24) and combining it with (9) we can obtain the corresponding exact solutions for (19).

$$
\text { When } \lambda^{2}-4 \mu>0 \text {, }
$$

$$
\begin{array}{r}
u_{1}(x, y, t) \\
=2 k l \mu-\frac{k l \lambda^{2}}{2}+\frac{k l\left(\lambda^{2}-4 \mu\right)}{2} \\
\times\left(\left(C_{1} \sinh \frac{\sqrt{\lambda^{2}-4 \mu}}{2} \xi\right.\right. \\
\left.+C_{2} \cosh \frac{\sqrt{\lambda^{2}-4 \mu}}{2} \xi\right) \\
\times\left(C_{1} \cosh \frac{\sqrt{\lambda^{2}-4 \mu}}{2} \xi\right. \\
\left.\left.+C_{2} \sinh \frac{\sqrt{\lambda^{2}-4 \mu}}{2} \xi\right)^{-1}\right)^{2},
\end{array}
$$

$$
\begin{array}{r}
v_{1}(x, y, t) \\
=2 k^{2} \mu-\frac{k^{2} \lambda^{2}}{2}+\frac{k^{2}}{2}\left(\lambda^{2}-4 \mu\right) \\
\times\left(\left(C_{1} \sinh \frac{\sqrt{\lambda^{2}-4 \mu}}{2} \xi\right.\right. \\
\left.+C_{2} \cosh \frac{\sqrt{\lambda^{2}-4 \mu}}{2} \xi\right)
\end{array}
$$

$$
\begin{gathered}
\times\left(C_{1} \cosh \frac{\sqrt{\lambda^{2}-4 \mu}}{2} \xi\right. \\
\left.\left.+C_{2} \sinh \frac{\sqrt{\lambda^{2}-4 \mu}}{2} \xi\right)^{-1}\right)^{2},
\end{gathered}
$$

$w_{1}(x, y, t)$

$$
=2 l^{2} \mu-\frac{l^{2} \lambda^{2}}{2}+\frac{l^{2}}{2}\left(\lambda^{2}-4 \mu\right)
$$

$$
\times\left(\left(C_{1} \sinh \frac{\sqrt{\lambda^{2}-4 \mu}}{2} \xi\right.\right.
$$

$$
\left.+C_{2} \cosh \frac{\sqrt{\lambda^{2}-4 \mu}}{2} \xi\right)
$$

$$
\begin{aligned}
& \times\left(C_{1} \cosh \frac{\sqrt{\lambda^{2}-4 \mu}}{2} \xi\right. \\
& \left.\left.+C_{2} \sinh \frac{\sqrt{\lambda^{2}-4 \mu}}{2} \xi\right)^{-1}\right)^{2},
\end{aligned}
$$

where $\xi=\left(\left(4 a k^{3} \mu-a k^{3} \lambda^{2}-b l^{3} \lambda^{2}-d l+4 b l^{3} \mu-c k\right) / \Gamma(1+\right.$ $\alpha)) t^{\alpha}+(k / \Gamma(1+\beta)) x^{\beta}+(l / \Gamma(1+\gamma)) y^{\gamma}+\xi_{0}$.

When $\lambda^{2}-4 \mu<0$,

$$
\begin{aligned}
& u_{2}(x, y, t) \\
& =2 k l \mu-\frac{k l \lambda^{2}}{2}+\frac{k l\left(4 \mu-\lambda^{2}\right)}{2} \\
& \times\left(\left(-C_{1} \sin \frac{\sqrt{4 \mu-\lambda^{2}}}{2} \xi\right.\right. \\
& \left.+C_{2} \cos \frac{\sqrt{4 \mu-\lambda^{2}}}{2} \xi\right) \\
& \times\left(C_{1} \cos \frac{\sqrt{4 \mu-\lambda^{2}}}{2} \xi\right. \\
& \left.\left.+C_{2} \sin \frac{\sqrt{4 \mu-\lambda^{2}}}{2} \xi\right)^{-1}\right)^{2},
\end{aligned}
$$




$$
\begin{array}{r}
v_{2}(x, y, t) \\
=2 k^{2} \mu-\frac{k^{2} \lambda^{2}}{2}+\frac{k^{2}}{2}\left(4 \mu-\lambda^{2}\right) \\
\times\left(\left(-C_{1} \sin \frac{\sqrt{4 \mu-\lambda^{2}}}{2} \xi\right.\right. \\
\left.+C_{2} \cos \frac{\sqrt{4 \mu-\lambda^{2}}}{2} \xi\right) \\
\times\left(C_{1} \cos \frac{\sqrt{4 \mu-\lambda^{2}}}{2} \xi\right. \\
\left.\left.+C_{2} \sin \frac{\sqrt{4 \mu-\lambda^{2}}}{2} \xi\right)^{-1}\right)^{2},
\end{array}
$$$$
w_{2}(x, y, t)
$$$$
=2 l^{2} \mu-\frac{l^{2} \lambda^{2}}{2}+\frac{l^{2}}{2}\left(4 \mu-\lambda^{2}\right)
$$$$
\times\left(\left(-C_{1} \sin \frac{\sqrt{4 \mu-\lambda^{2}}}{2} \xi\right.\right.
$$$$
\left.+C_{2} \cos \frac{\sqrt{4 \mu-\lambda^{2}}}{2} \xi\right)
$$$$
\times\left(C_{1} \cos \frac{\sqrt{4 \mu-\lambda^{2}}}{2} \xi\right.
$$$$
\left.\left.+C_{2} \sin \frac{\sqrt{4 \mu-\lambda^{2}}}{2} \xi\right)^{-1}\right)^{2},
$$

where $\xi=\left(\left(4 a k^{3} \mu-a k^{3} \lambda^{2}-b l^{3} \lambda^{2}-d l+4 b l^{3} \mu-c k\right) / \Gamma(1+\right.$ $\alpha)) t^{\alpha}+(k / \Gamma(1+\beta)) x^{\beta}+(l / \Gamma(1+\gamma)) y^{\gamma}+\xi_{0}$.

When $\lambda^{2}-4 \mu=0$,

$$
\begin{aligned}
& u_{3}(x, y, t)=-\frac{k l \lambda^{2}}{2}+\frac{2 k l C_{2}^{2}}{\left(C_{1}+C_{2} \xi\right)^{2}}+2 k l \mu, \\
& v_{3}(x, y, t)=-\frac{k^{2} \lambda^{2}}{2}+\frac{2 k^{2} C_{2}^{2}}{\left(C_{1}+C_{2} \xi\right)^{2}}+2 k^{2} \mu, \\
& w_{3}(x, y, t)=-\frac{l^{2} \lambda^{2}}{2}+\frac{2 l^{2} C_{2}^{2}}{\left(C_{1}+C_{2} \xi\right)^{2}}+2 l^{2} \mu,
\end{aligned}
$$

where $\xi=\left(\left(4 a k^{3} \mu-a k^{3} \lambda^{2}-b l^{3} \lambda^{2}-d l+4 b l^{3} \mu-c k\right) / \Gamma(1+\right.$ $\alpha)) t^{\alpha}+(k / \Gamma(1+\beta)) x^{\beta}+(l / \Gamma(1+\gamma)) y^{\gamma}+\xi_{0}$.

Case 2. Consider

$$
\begin{gathered}
a_{2}=2 k l, \quad a_{1}=2 k l \lambda, \quad a_{0}=\frac{1}{3} k l\left(\lambda^{2}+2 \mu\right), \\
b_{2}=2 k^{2}, \quad b_{1}=2 k^{2} \lambda, \quad b_{0}=\frac{1}{3} k^{2}\left(\lambda^{2}+2 \mu\right), \\
c_{2}=2 l^{2}, \quad c_{1}=2 l^{2} \lambda, \quad c_{0}=\frac{1}{3} l^{2}\left(\lambda^{2}+2 \mu\right), \\
c=-4 a k^{3} \mu+a k^{3} \lambda^{2}+b l^{3} \lambda^{2}-d l-4 b l^{3} \mu-c k, \\
g_{1}=g_{2}=g_{3}=0 .
\end{gathered}
$$

Substituting the result above into (24) and combining it with (9) we can obtain the corresponding exact solutions for (19).

When $\lambda^{2}-4 \mu>0$,

$$
\begin{array}{r}
u_{4}(x, y, t) \\
=\frac{1}{3} k l\left(\lambda^{2}+2 \mu\right)-\frac{k l \lambda^{2}}{2}+\frac{k l\left(\lambda^{2}-4 \mu\right)}{2} \\
\times\left(\left(C_{1} \sinh \frac{\sqrt{\lambda^{2}-4 \mu}}{2} \xi\right.\right. \\
\left.+C_{2} \cosh \frac{\sqrt{\lambda^{2}-4 \mu}}{2} \xi\right) \\
\times\left(C_{1} \cosh \frac{\sqrt{\lambda^{2}-4 \mu}}{2} \xi\right. \\
\left.\left.+C_{2} \sinh \frac{\sqrt{\lambda^{2}-4 \mu}}{2} \xi\right)^{-1}\right)^{2},
\end{array}
$$

$$
\begin{aligned}
& v_{4}(x, y, t) \\
& =\frac{1}{3} k^{2}\left(\lambda^{2}+2 \mu\right)-\frac{k^{2} \lambda^{2}}{2}+\frac{k^{2}}{2}\left(\lambda^{2}-4 \mu\right) \\
& \times\left(\left(C_{1} \sinh \frac{\sqrt{\lambda^{2}-4 \mu}}{2} \xi\right.\right. \\
& \left.+C_{2} \cosh \frac{\sqrt{\lambda^{2}-4 \mu}}{2} \xi\right)
\end{aligned}
$$




$$
\begin{aligned}
& \times\left(C_{1} \cosh \frac{\sqrt{\lambda^{2}-4 \mu}}{2} \xi\right. \\
& \left.\left.+C_{2} \sinh \frac{\sqrt{\lambda^{2}-4 \mu}}{2} \xi\right)^{-1}\right)^{2}, \\
& w_{4}(x, y, t x i) \\
& =\frac{1}{3} l^{2}\left(\lambda^{2}+2 \mu\right)-\frac{l^{2} \lambda^{2}}{2}+\frac{l^{2}}{2}\left(\lambda^{2}-4 \mu\right) \\
& \times\left(\left(C_{1} \sinh \frac{\sqrt{\lambda^{2}-4 \mu}}{2} \xi\right.\right. \\
& \left.+C_{2} \cosh \frac{\sqrt{\lambda^{2}-4 \mu}}{2} \xi\right) \\
& \times\left(C_{1} \cosh \frac{\sqrt{\lambda^{2}-4 \mu}}{2} \xi\right. \\
& \left.\left.+C_{2} \sinh \frac{\sqrt{\lambda^{2}-4 \mu}}{2} \xi\right)^{-1}\right)^{2},
\end{aligned}
$$

where $\xi=\left(\left(-4 a k^{3} \mu+a k^{3} \lambda^{2}+b l^{3} \lambda^{2}-d l-4 b l^{3} \mu-c k\right) / \Gamma(1+\right.$ $\alpha)) t^{\alpha}+(k / \Gamma(1+\beta)) x^{\beta}+(l / \Gamma(1+\gamma)) y^{\gamma}+\xi_{0}$.

When $\lambda^{2}-4 \mu<0$,

$$
\begin{aligned}
& u_{5}(x, y, t) \\
& =\frac{1}{3} k l\left(\lambda^{2}+2 \mu\right)-\frac{k l \lambda^{2}}{2}+\frac{k l\left(4 \mu-\lambda^{2}\right)}{2} \\
& \times\left(\left(-C_{1} \sin \frac{\sqrt{4 \mu-\lambda^{2}}}{2} \xi\right.\right. \\
& \left.+C_{2} \cos \frac{\sqrt{4 \mu-\lambda^{2}}}{2} \xi\right) \\
& \times\left(C_{1} \cos \frac{\sqrt{4 \mu-\lambda^{2}}}{2} \xi\right. \\
& \left.\left.+C_{2} \sin \frac{\sqrt{4 \mu-\lambda^{2}}}{2} \xi\right)^{-1}\right)^{2}
\end{aligned}
$$

$$
\begin{array}{r}
v_{5}(x, y, t) \\
=\frac{1}{3} k^{2}\left(\lambda^{2}+2 \mu\right)-\frac{k^{2} \lambda^{2}}{2}+\frac{k^{2}}{2}\left(4 \mu-\lambda^{2}\right) \\
\times\left(\left(-C_{1} \sin \frac{\sqrt{4 \mu-\lambda^{2}}}{2} \xi\right.\right. \\
\left.+C_{2} \cos \frac{\sqrt{4 \mu-\lambda^{2}}}{2} \xi\right) \\
\times\left(C_{1} \cos \frac{\sqrt{4 \mu-\lambda^{2}}}{2} \xi\right. \\
\left.\left.+C_{2} \sin \frac{\sqrt{4 \mu-\lambda^{2}}}{2} \xi\right)^{-1}\right)^{2},
\end{array}
$$

$w_{5}(x, y, t)$

$$
\begin{aligned}
= & \frac{1}{3} l^{2}\left(\lambda^{2}+2 \mu\right)-\frac{l^{2} \lambda^{2}}{2}+\frac{l^{2}}{2}\left(4 \mu-\lambda^{2}\right) \\
& \times\left(\left(-C_{1} \sin \frac{\sqrt{4 \mu-\lambda^{2}}}{2} \xi\right.\right.
\end{aligned}
$$

$$
\begin{gathered}
\left.+C_{2} \cos \frac{\sqrt{4 \mu-\lambda^{2}}}{2} \xi\right) \\
\times\left(C_{1} \cos \frac{\sqrt{4 \mu-\lambda^{2}}}{2} \xi\right. \\
\left.\left.+C_{2} \sin \frac{\sqrt{4 \mu-\lambda^{2}}}{2} \xi\right)^{-1}\right)^{2},
\end{gathered}
$$

where $\xi=\left(\left(-4 a k^{3} \mu+a k^{3} \lambda^{2}+b l^{3} \lambda^{2}-d l-4 b l^{3} \mu-c k\right) / \Gamma(1+\right.$ $\alpha)) t^{\alpha}+(k / \Gamma(1+\beta)) x^{\beta}+(l / \Gamma(1+\gamma)) y^{\gamma}+\xi_{0}$.

When $\lambda^{2}-4 \mu=0$,

$$
\begin{aligned}
& u_{6}(x, y, t)=-\frac{k l \lambda^{2}}{2}+\frac{2 k l C_{2}^{2}}{\left(C_{1}+C_{2} \xi\right)^{2}}+\frac{1}{3} k l\left(\lambda^{2}+2 \mu\right), \\
& v_{6}(x, y, t)=-\frac{k^{2} \lambda^{2}}{2}+\frac{2 k^{2} C_{2}^{2}}{\left(C_{1}+C_{2} \xi\right)^{2}}+\frac{1}{3} k^{2}\left(\lambda^{2}+2 \mu\right), \\
& w_{6}(x, y, t)=-\frac{l^{2} \lambda^{2}}{2}+\frac{2 l^{2} C_{2}^{2}}{\left(C_{1}+C_{2} \xi\right)^{2}}+\frac{1}{3} l^{2}\left(\lambda^{2}+2 \mu\right),
\end{aligned}
$$


where $\xi=\left(\left(-4 a k^{3} \mu+a k^{3} \lambda^{2}+b l^{3} \lambda^{2}-d l-4 b l^{3} \mu-c k\right) / \Gamma(1+\right.$ $\alpha)) t^{\alpha}+(k / \Gamma(1+\beta)) x^{\beta}+(l / \Gamma(1+\gamma)) y^{\gamma}+\xi_{0}$.

Remark 2. The established solutions in (26)-(32) for the space-time fractional $(2+1)$-dimensional Nizhnik-NovikovVeselov system have not been reported by other authors so far.

Remark 3. In [21], Zayed solved the $(2+1)$-dimensional Nizhnik-Novikov-Veselov (NNV) system $(20)$ by the $\left(G^{\prime} / G\right)$ expansion method. We note that if we put $\alpha=1, k=l=1$ in (26)-(32), then our results reduce to the results in [21] despite the slight difference of the symbols. So the solutions presented here are of more general forms.

3.3. Time Fractional Fifth-Order Sawada-Kotera Equation. We consider the time fractional fifth-order Sawada-Kotera equation:

$$
\begin{aligned}
D_{t}^{\alpha} u & +u_{x x x x x}+45 u_{x} u^{2} \\
& +15\left(u_{x} u_{x x}+u u_{x x x}\right)=0, \quad 0<\alpha \leq 1,
\end{aligned}
$$

which is the variation of the fifth-order Sawada-Kotera equation [39]:

$$
u_{t}+u_{x x x x x}+45 u_{x} u^{2}+15\left(u_{x} u_{x x}+u u_{x x x}\right)=0 .
$$

Now we apply the proposed method to (33). To begin with, we suppose $u(x, t)=U(\xi)$, where $\xi=k x+(c / \Gamma(1+\alpha)) t^{\alpha}+\xi_{0}$, $k, c, \xi_{0}$ are all constants with $k, c \neq 0$. Then by the use of the first equality in (3), (33) can be turned into

$$
c U^{\prime}+k^{5} U^{(5)}+45 k U U^{\prime}+15 k^{3}\left(U^{\prime} U^{\prime \prime}+U U^{\prime \prime \prime}\right)=0 .
$$

Suppose that the solution of (35) can be expressed by

$$
U(\xi)=\sum_{i=0}^{m} a_{i}\left(\frac{G^{\prime}}{G}\right)^{i} .
$$

Balancing the order of $U^{(5)}$ and $U U^{\prime \prime \prime}$ in (36) we have $m=2$. So

$$
U(\xi)=a_{0}+a_{1}\left(\frac{G^{\prime}}{G}\right)+a_{2}\left(\frac{G^{\prime}}{G}\right)^{2} .
$$

Substituting (37) into (35), using (8), collecting all the terms with the same power of $\left(G^{\prime} / G\right)$ together, and equating each coefficient to zero, yields a set of algebraic equations. Solving these equations yields

$$
\begin{aligned}
& a_{0}=-\left(\left(40 k^{3} \mu+5 k^{3} \lambda^{2}\right.\right. \\
& \left.\quad \pm \sqrt{80 k^{6} \mu^{2}-40 k^{6} \mu \lambda^{2}+5 k^{6} \lambda^{4}-20 k c}\right) \\
& \left.\times(30 k)^{-1}\right), \\
& a_{1}=-2 k^{2} \lambda, \quad a_{2}=-2 k^{2} .
\end{aligned}
$$

Substituting the result above into (37) and combining it with (9) we can obtain the corresponding exact solutions to (33).

$$
\begin{aligned}
& \text { When } \lambda^{2}-4 \mu>0 \text {, } \\
& u_{1}(x, t) \\
& =-\left(\left(40 k^{3} \mu+5 k^{3} \lambda^{2}\right.\right. \\
& \left. \pm \sqrt{80 k^{6} \mu^{2}-40 k^{6} \mu \lambda^{2}+5 k^{6} \lambda^{4}-20 k c}\right) \\
& \left.\times(30 k)^{-1}\right) \\
& -2 k^{2} \lambda\left[-\frac{\lambda}{2}+\frac{\sqrt{\lambda^{2}-4 \mu}}{2}\right. \\
& \times\left(\left(C_{1} \sinh \frac{\sqrt{\lambda^{2}-4 \mu}}{2} \xi\right.\right. \\
& \left.+C_{2} \cosh \frac{\sqrt{\lambda^{2}-4 \mu}}{2} \xi\right) \\
& \times\left(C_{1} \cosh \frac{\sqrt{\lambda^{2}-4 \mu}}{2} \xi\right. \\
& \left.\left.\left.+C_{2} \sinh \frac{\sqrt{\lambda^{2}-4 \mu}}{2} \xi\right)^{-1}\right)\right]
\end{aligned}
$$$$
-2 k^{2}\left[-\frac{\lambda}{2}+\frac{\sqrt{\lambda^{2}-4 \mu}}{2}\right.
$$

$$
\begin{gathered}
\times\left(\left(C_{1} \sinh \frac{\sqrt{\lambda^{2}-4 \mu}}{2} \xi\right.\right. \\
\left.+C_{2} \cosh \frac{\sqrt{\lambda^{2}-4 \mu}}{2} \xi\right) \\
\times\left(C_{1} \cosh \frac{\sqrt{\lambda^{2}-4 \mu}}{2} \xi\right. \\
\left.\left.\left.+C_{2} \sinh \frac{\sqrt{\lambda^{2}-4 \mu}}{2} \xi\right)^{-1}\right)\right]^{2},
\end{gathered}
$$

where $\xi=k x+(c / \Gamma(1+\alpha)) t^{\alpha}+\xi_{0}$. 


$$
\begin{aligned}
& \text { When } \lambda^{2}-4 \mu<0 \text {, } \\
& u_{2}(x, t) \\
& =-\left(\left(40 k^{3} \mu+5 k^{3} \lambda^{2}\right.\right. \\
& \left. \pm \sqrt{80 k^{6} \mu^{2}-40 k^{6} \mu \lambda^{2}+5 k^{6} \lambda^{4}-20 k c}\right) \\
& \times(30 k))^{-1} \\
& -2 k^{2} \lambda\left[-\frac{\lambda}{2}+\frac{\sqrt{4 \mu-\lambda^{2}}}{2}\right. \\
& \times\left(\left(-C_{1} \sin \frac{\sqrt{4 \mu-\lambda^{2}}}{2} \xi\right.\right. \\
& \left.+C_{2} \cos \frac{\sqrt{4 \mu-\lambda^{2}}}{2} \xi\right) \\
& \times\left(C_{1} \cos \frac{\sqrt{4 \mu-\lambda^{2}}}{2} \xi\right. \\
& \left.\left.\left.+C_{2} \sin \frac{\sqrt{4 \mu-\lambda^{2}}}{2} \xi\right)^{-1}\right)\right] \\
& -2 k^{2}\left[-\frac{\lambda}{2}+\frac{\sqrt{4 \mu-\lambda^{2}}}{2}\right. \\
& \times\left(\left(-C_{1} \sin \frac{\sqrt{4 \mu-\lambda^{2}}}{2} \xi\right.\right. \\
& \left.+C_{2} \cos \frac{\sqrt{4 \mu-\lambda^{2}}}{2} \xi\right) \\
& \times\left(C_{1} \cos \frac{\sqrt{4 \mu-\lambda^{2}}}{2} \xi\right. \\
& \left.\left.\left.+C_{2} \sin \frac{\sqrt{4 \mu-\lambda^{2}}}{2} \xi\right)^{-1}\right)\right]^{2}
\end{aligned}
$$

where $\xi=k x+(c / \Gamma(1+\alpha)) t^{\alpha}+\xi_{0}$.
When $\lambda^{2}-4 \mu=0$,

$$
\begin{aligned}
& u_{3}(x, t) \\
& =-\left(\left(40 k^{3} \mu+5 k^{3} \lambda^{2}\right.\right. \\
& \left. \pm \sqrt{80 k^{6} \mu^{2}-40 k^{6} \mu \lambda^{2}+5 k^{6} \lambda^{4}-20 k c}\right) \\
& \left.\times(30 k)^{-1}\right) \\
& -2 k^{2} \lambda\left[-\frac{\lambda}{2}+\frac{C_{2}}{C_{1}+C_{2} \xi}\right] \\
& -2 k^{2}\left[-\frac{\lambda}{2}+\frac{C_{2}}{C_{1}+C_{2} \xi}\right]^{2}
\end{aligned}
$$

where $\xi=k x+(c / \Gamma(1+\alpha)) t^{\alpha}+\xi_{0}$.

Remark 4. The established solutions in (39)-(41) are new exact solutions for the time fractional fifth-order SawadaKotera equation.

\section{Conclusions}

We have solved the space-time fractional $(2+1)$-dimensional dispersive long wave equations, the space-time fractional $(2+1)$-dimensional Nizhnik-Novikov-Veselov system, and the time fractional fifth-order Sawada-Kotera equation by the known $\left(G^{\prime} / G\right)$ method successfully. In the process, the transformation for $\xi$ is very important, which ensures that certain fractional partial differential equations can be turned into ordinary differential equations of integer order. With the aid of mathematical software, a variety of exact solutions for these equations are obtained, which may help to understand better physical phenomenon related to these equations. Finally, we note that this approach can also be applied to solve other fractional partial differential equations.

\section{Conflict of Interests}

The author declares that there is no conflict of interests regarding the publication of this paper.

\section{References}

[1] G.-C. Wu, "A fractional variational iteration method for solving fractional nonlinear differential equations," Computers and Mathematics with Applications, vol. 61, no. 8, pp. 2186-2190, 2011.

[2] J. Ji, J. B. Zhang, and Y. J. Dong, “The fractional variational iteration method improved with the Adomian series," Applied Mathematics Letters, vol. 25, pp. 2223-2226, 2012.

[3] G.-C. Wu and E. W. M. Lee, "Fractional variational iteration method and its application," Physics Letters A, vol. 374, no. 25, pp. 2506-2509, 2010.

[4] S. Guo and L. Mei, "The fractional variational iteration method using He's polynomials," Physics Letters A, vol. 375, no. 3, pp. 309-313, 2011. 
[5] M. Merdan, "A numeric-analytic method for time-fractional Swift-Hohenberg (S-H) equation with modified RiemannLiouville derivative," Applied Mathematical Modelling, vol. 37, pp. 4224-4231, 2013.

[6] A. M. A. El-Sayed, S. H. Behiry, and W. E. Raslan, "Adomian's decomposition method for solving an intermediate fractional advection-dispersion equation," Computers and Mathematics with Applications, vol. 59, no. 5, pp. 1759-1765, 2010.

[7] A. M. A. El-Sayed and M. Gaber, “The Adomian decomposition method for solving partial differential equations of fractal order in finite domains," Physics Letters A, vol. 359, no. 3, pp. 175-182, 2006.

[8] S. S. Ray, "A new approach for the application of Adomian decomposition method for the solution of fractional space diffusion equation with insulated ends," Applied Mathematics and Computation, vol. 202, no. 2, pp. 544-549, 2008.

[9] K. A. Gepreel, “The homotopy perturbation method applied to the nonlinear fractional KolmogorovPetrovskiiPiskunov equations," Applied Mathematics Letters, vol. 24, no. 8, pp. 1428-1434, 2011.

[10] M. Cui, "Compact finite difference method for the fractional diffusion equation," Journal of Computational Physics, vol. 228, no. 20, pp. 7792-7804, 2009.

[11] Q. Huang, G. Huang, and H. Zhan, "A finite element solution for the fractional advection-dispersion equation," Advances in Water Resources, vol. 31, no. 12, pp. 1578-1589, 2008.

[12] B. Lu, "Bäcklund transformation of fractional Riccati equation and its applications to nonlinear fractional partial differential equations," Physics Letters A, vol. 376, pp. 2045-2048, 2012.

[13] S. M. Guo, L. Q. Mei, Y. Li, and Y. F. Sun, “The improved fractional sub-equation method and its applications to the space-time fractional differential equations in fluid mechanics," Physics Letters A, vol. 376, no. 4, pp. 407-411, 2012.

[14] S. Zhang and H.-Q. Zhang, "Fractional sub-equation method and its applications to nonlinear fractional PDEs," Physics Letters A, vol. 375, no. 7, pp. 1069-1073, 2011.

[15] M. L. Wang, X. Z. Li, and J. L. Zhang, "The (frac(G', G))expansion method and travelling wave solutions of nonlinear evolution equations in mathematical physics," Physics Letters A, vol. 372, no. 4, pp. 417-423, 2008.

[16] B. Zheng, "(G'/G)-Expansion method for solving fractional partial differential equations in the theory of mathematical physics," Communications in Theoretical Physics, vol. 58, pp. 623-630, 2012.

[17] I. Aslan, "Discrete exact solutions to some nonlinear differential-difference equations via the (G'G)-expansion method," Applied Mathematics and Computation, vol. 215, no. 8, pp. 3140-3147, 2009.

[18] B. Ayhan and A. Bekir, "The (G'/G)-expansion method for the nonlinear lattice equations," Communications in Nonlinear Science and Numerical Simulation, vol. 17, pp. 3490-3498, 2012.

[19] K. A. Gepreel and S. Omran, "Exact solutions for nonlinear partial fractional differential equations," Chinese Physics B, vol. 21, no. 11, Article ID 110204, 7 pages, 2012.

[20] B. Zheng and C. Wen, "Exact solutions for fractional partial differential equations by a new fractional subequationmethod," Advances in Difference Equations, vol. 2013, article 199, 2013.

[21] E. M. E. Zayed, “The (G'G)-expansion method and its applications to some nonlinear evolution equations in the mathematical physics," Journal of Applied Mathematics and Computing, vol. 30, no. 1-2, pp. 89-103, 2009.
[22] G. Jumarie, "Modified Riemann-Liouville derivative and fractional Taylor series of nondifferentiable functions further results," Computers and Mathematics with Applications, vol. 51, no. 9-10, pp. 1367-1376, 2006.

[23] C. C. Kong, D. Wang, L. N. Song, and H. Q. Zhang, "New exact solutions to MKDV-Burgers equation and $(2+1)$-dimensional dispersive long wave equation via extended Riccati equation method," Chaos, Solitons and Fractals, vol. 39, no. 2, pp. 697706, 2009.

[24] Z. Y. Yan, "Generalized transformations and abundant new families of exact solutions for (2+1)-dimensional dispersive long wave equations," Computers and Mathematics with Applications, vol. 46, no. 8-9, pp. 1363-1372, 2003.

[25] J. Zhang and P. Han, "New multisoliton solutions of the (2+1)dimensional dispersive long wave equations," Communications in Nonlinear Science and Numerical Simulation, vol. 6, no. 3, pp. 178-182, 2001.

[26] M. Eslami, A. Neyrame, and M. Ebrahimi, "Explicit solutions of nonlinear (2+1)-dimensional dispersive long wave equation," Journal of King Saud University—Science, vol. 24, no. 1, pp. 6971, 2012.

[27] Y. Chen and Q. Wang, "A new general algebraic method with symbolic computation to construct new doubly-periodic solutions of the (2+1)-dimensional dispersive long wave equation," Applied Mathematics and Computation, vol. 167, no. 2, pp. 919929, 2005.

[28] Q. Wang, Y. Chen, and H. Q. Zhang, "An extended Jacobi elliptic function rational expansion method and its application to $(2+1)$-dimensional dispersive long wave equation," Physics Letters A, vol. 340, no. 5-6, pp. 411-426, 2005.

[29] Y. Chen and B. Li, "Symbolic computation and construction of soliton-like solutions to the (2+1)-dimensional dispersive longwave equations," International Journal of Engineering Science, vol. 42, no. 7, pp. 715-724, 2004.

[30] N. Liu, X. Q. Liu, and H. L. Lu, "New exact solutions and conservation laws of the (2+1)-dimensional dispersive long wave equations," Physics Letters A, vol. 373, no. 2, pp. 214-220, 2009.

[31] S. Zhang, J.-L. Tong, and W. Wang, "Exp-function method for a nonlinear ordinary differential equation and new exact solutions of the dispersive long wave equations," Computers and Mathematics with Applications, vol. 58, no. 11-12, pp. 2294-2299, 2009.

[32] Y. Q. Zhou, Q. Liu, J. Zhang, and W. N. Zhang, "Exact solution for (2+1)-dimension nonlinear dispersive long wave equation," Applied Mathematics and Computation, vol. 177, no. 2, pp. 495499, 2006.

[33] E. Yomba, “The modified extended Fan's sub-equation method and its application to $(2+1)$-dimensional dispersive long wave equation," Chaos, Solitons and Fractals, vol. 26, no. 3, pp. 785794, 2005.

[34] Y. Chen and Q. Wang, "A series of new soliton-like solutions and double-like periodic solutions of a (2+1)-dimensional dispersive long wave equation," Chaos, Solitons and Fractals, vol. 23, no. 3, pp. 801-807, 2005.

[35] X. Zeng and J. Zeng, "Symbolic computation and new families of exact solutions to the $(2+1)$-dimensional dispersive longwave equations," Chaos, Solitons and Fractals, vol. 29, no. 5, pp. 1115-1120, 2006.

[36] Z. Sheng, "The periodic wave solutions for the $(2+1)$ dimensional dispersive long water equations," Chaos, Solitons and Fractals, vol. 32, no. 2, pp. 847-854, 2007. 
[37] A.-M. Wazwaz, "New solitary wave and periodic wave solutions to the (2+1)-dimensional Nizhnik-Novikov-Veselov system," Applied Mathematics and Computation, vol. 187, no. 2, pp. 15841591, 2007.

[38] C. Senthil Kumar, R. Radha, and M. Lakshmanan, "Trilinearization and localized coherent structures and periodic solutions for the $(2+1)$ dimensional K-dV and NNV equations," Chaos, Solitons and Fractals, vol. 39, no. 2, pp. 942-955, 2009.

[39] C. Liu and Z. Dai, "Exact soliton solutions for the fifth-order Sawada-Kotera equation," Applied Mathematics and Computation, vol. 206, no. 1, pp. 272-275, 2008. 


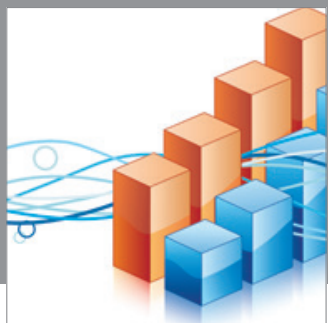

Advances in

Operations Research

mansans

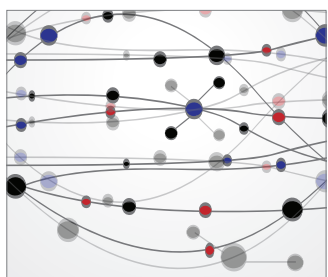

The Scientific World Journal
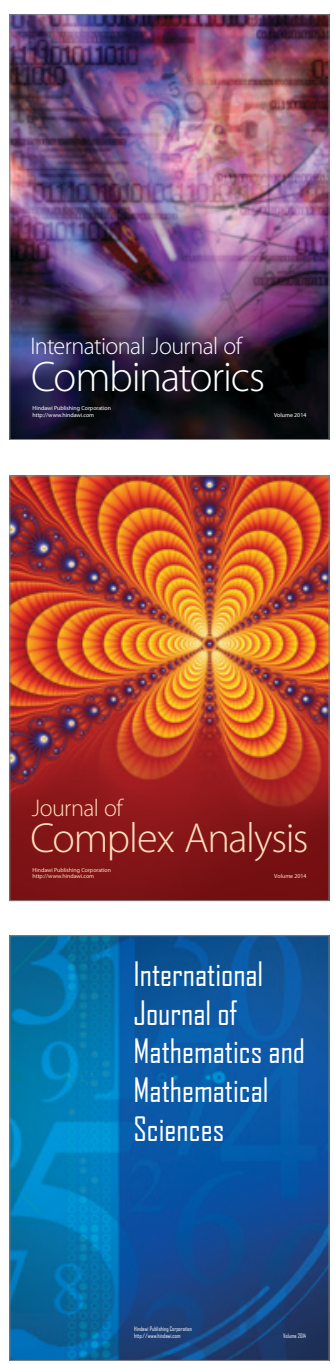
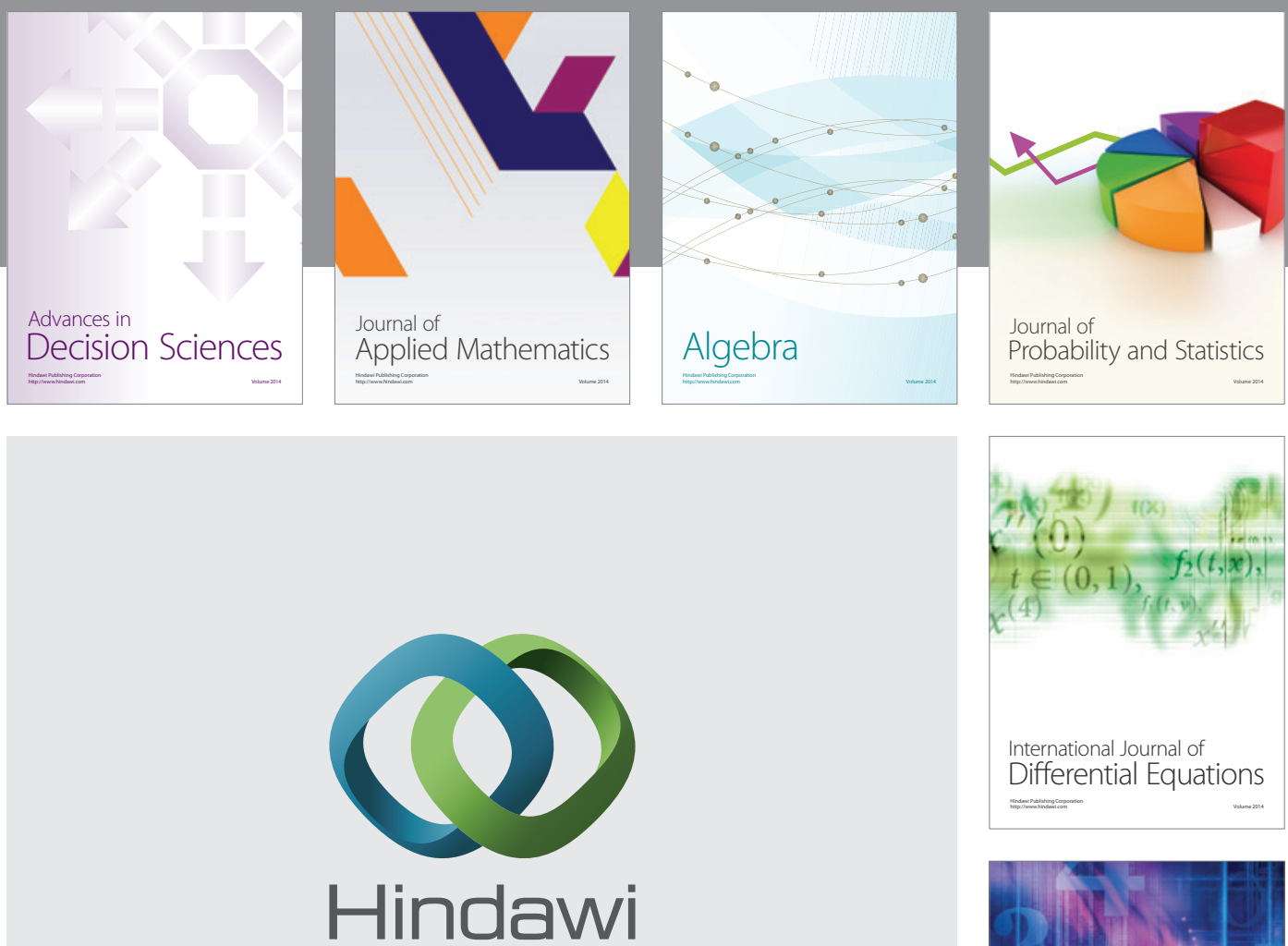

Submit your manuscripts at http://www.hindawi.com
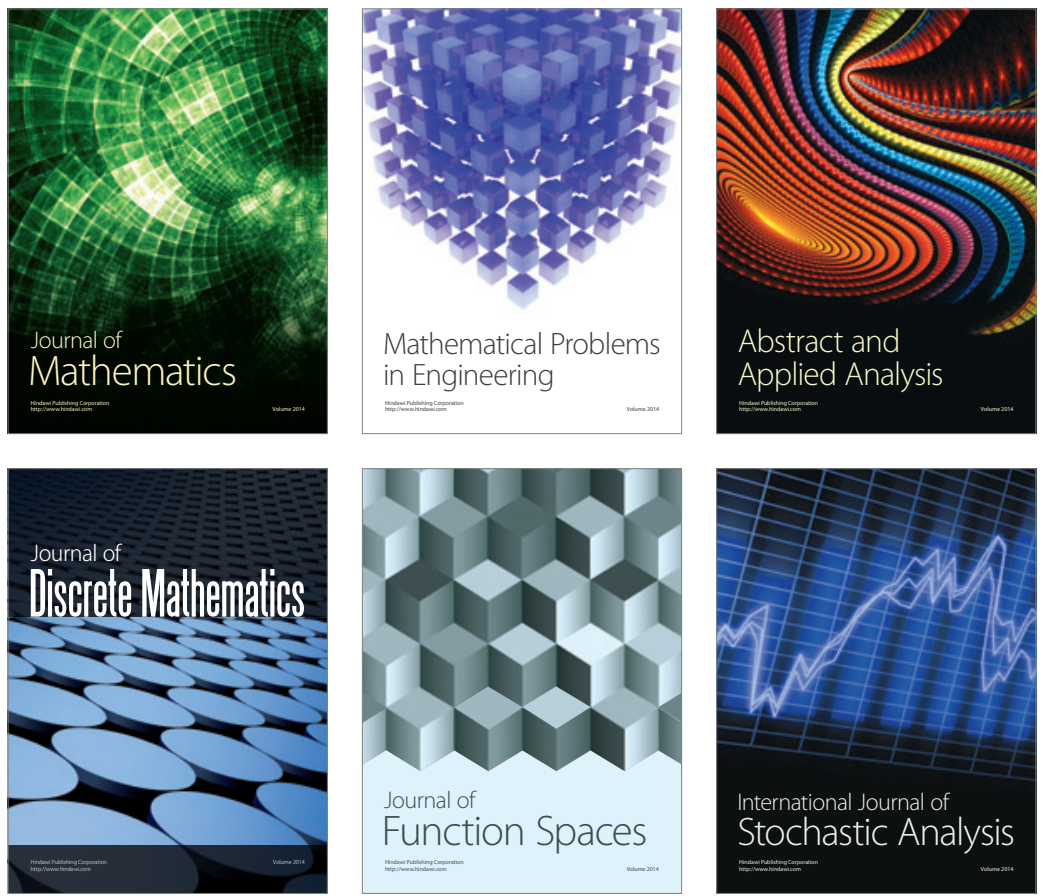

Journal of

Function Spaces

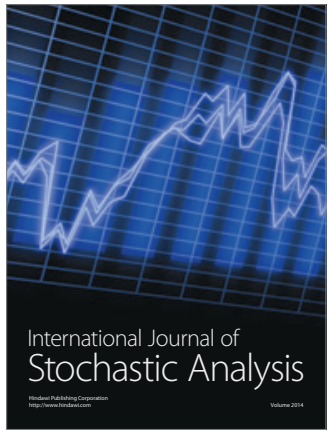

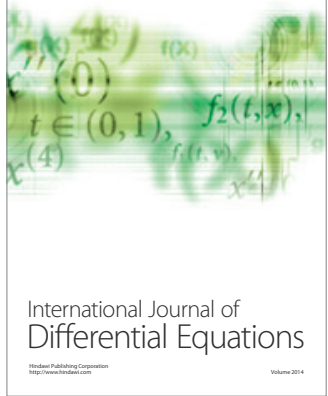
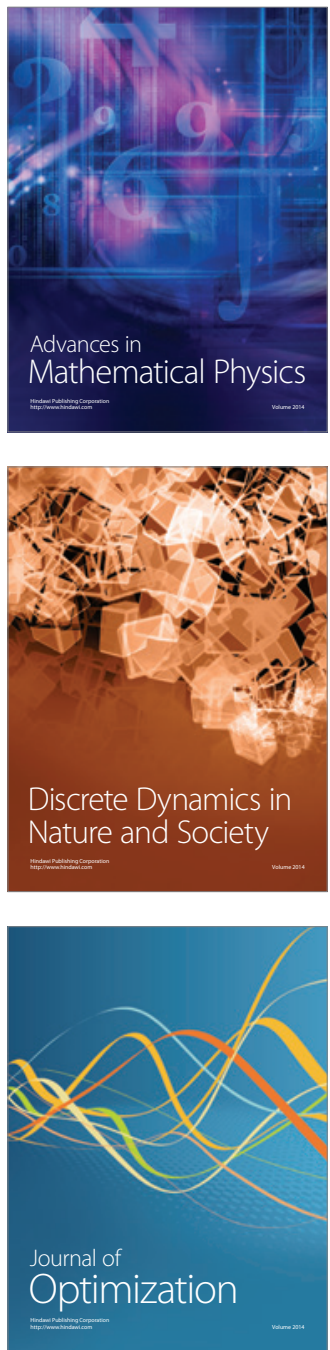